\title{
The TasLab portal for collaborative innovation
}

\author{
Pavel Shvaiko ${ }^{1}$, Luca Mion ${ }^{1}$, Fabiano Dalpiaz ${ }^{2}$ and Giuseppe Angelini ${ }^{3}$ \\ ${ }^{1}$ TasLab, Informatica Trentina, via Gilli 2, 38121, Trento, Italy \\ \{pavel.shvaiko,luca.mion\}@infotn.it \\ ${ }^{2}$ DISI, University of Trento, via Sommarive 14, 38123, Trento, Italy \\ fabiano.dalpiaz@disi.it \\ ${ }^{3}$ WEBSS srl, Web Software Solutions, via Alto Adige 170, 38121, Trento, Italy \\ gangelini@webss.it
}

\begin{abstract}
In this paper we present our experience with fostering collaborative innovation within the Trentino as a Lab (TasLab) environment, by creating the conditions for a successful integration of the innovation stakeholders in Trentino, Italy. In order to technically foster innovation we have implemented the TasLab portal, with the aim of promoting the synergic coordination and technology transfer among the three main innovation stakeholders that form the so-called innovation tripole: (i) final users, bringing domain knowledge and expectations, (ii) enterprises and SMEs, bringing knowledge of the market, and (iii) research centres, bringing the latest research results. The portal provides various functionalities related to collaborative innovation, including semi-automatic innovation tripole generation from the user created content, semantic search and so on. Finally, we discuss some implications of the approach implemented on the body of knowledge on innovation and concurrent enterprising.
\end{abstract}

Keywords

Collaborative innovation engineering, web portals, semantic technology.

\section{Introduction}

The process of innovation in ICT services has traditionally been suffering from a little and late interaction with the actual market and users, causing private and public investments to fail or return little value. In order to address this problem, during the recent years industries are trying to adapt their organization by adopting an open innovation approach [Chesbrough, 2003; Gassmann \& Enkel, 2004], namely by bringing stakeholders to innovate through collaboration instead of handling all R\&D activities internally. This approach has reflected a more systematic paradigm shift from an organizational setting towards a consumer market, where end-users are involved in a way that the development of ICT services is performed around the actual users themselves. A major difference between such a co-creative process and the traditional innovation process is in terms of how the user is perceived. Specifically, instead of bringing the user in during the development phase (i.e., participatory design), the user is moved in the innovation process to a higher level - as a source of innovation.

This model is already adopted by Living Labs, which are open innovation environments in reallife settings where user-driven innovation is integrated within the co-creation process of new services, products and societal infrastructures. This concept has emerged and gained interest as it pursues the idea to further increase the role of the user in the innovation process, who is seen as a consumer, a citizen, part of a community in a close collaboration with market and technology [Eriksson et al., 2005]. Within this framework, Living Labs include a network of organisations and stakeholders involved in the co-creation process in combining technology push and application pull, where users co-create and validate services in real life settings in a number of domains, such as mobile ICT [de Leon et al., 2006] and collaborative work [Katzy, 2005]. 
Growing political interest in the subject demonstrates the importance of the Living Labs concept as a mechanism to strengthen European innovation performance: an initiative that supports such a collaboration approach is the foundation of the European Network of Living Labs ${ }^{1}$ (ENoLL), with the purpose of strengthening the innovative capabilities within the EU by promoting the territories as experimental facilities involving users throughout the innovation process. An important role is then expected to be played by Living Labs to help bridge the innovation gap between research (prototypes) and market (products/services), in order to help new technologies push on innovative services, allowing applications to benefit from an economy of scale. In these terms, public users (including governments and citizens) are to play a key role in the innovation scenario.

Living Labs are by definition multidisciplinary eco-systems structured around specific domains. As such, the co-operation among the stakeholders within Living Labs includes actors with different ways to exploit the transversal infrastructure (e.g., broadband or testebeds) and it includes users to be involved in the experimental facility, using services and methodologies in many different ways. In this context, tools that aim at supporting the management of innovation processes have to convey ideas and to supply suitable tools and operations applied to those ideas in order to manage the diversities that characterize the actors involved in the innovation process. Various tools have been developed in the past to create networks and to provide technical instruments to facilitate the activities of innovation stakeholders, supporting the different phases of idea generation and idea evaluation [Lausen et al., 2005; van Gundy, 1988; Ardilio et al., 2004]. A trend in innovation management also includes the implementation of openly accessible idea Web portals as a form of user innovation toolkits [von Hippel, 2005, Riedl et al., 2009]. Within such portals, the management of diversity relies completely on the experience of users of the portals and no functionalities are provided in order to support the users towards shared visions, strategies and to help find the right partners to build a business or project proposal. Moreover, diversity is often considered as an issue discouraging users to access such portals, hence affecting their effectiveness.

Instead, we aim at handling properly the diversity in order to create the conditions for a successful collaboration and interaction among the innovation stakeholders. In particular, the innovation framework where such a diversity has emerged and where we set the requirements for the portal is the Living Lab of Trentino. Unlike the traditional approach to innovation management that considers such a diversity as an issue, we believe that it is rather a value that should be handled properly in order to create the conditions for a successful collaboration [Giunchiglia, 2007]. Existing tools do not provide enough technical support such that the stakeholders can easily share their ideas at the conceptual level. In this paper we present the TasLab portal that provides knowledge management facilities (e.g., competence matching) for collaborative innovation. The portal aims not only to model the pre-existing knowledge, but also to foster collaboration within public administrations, industrial companies and research centres by suitably managing heterogeneity and diversity of these actors, e.g., with the functionality that semi-automatically generates the so-called innovation tripoles by performing a semantic search over the business partners registered on the portal.

The rest of the paper is organized as follows. In Section 2 we discuss the TasLab network and its specific features. Then, in Section 3 we overview some of the functionalities of the TasLab portal, which are related to collaborative innovation. Section 4 provides a discussion on some implications of the implemented approach on innovation and concurrent enterprising. Finally, Section 5 summarizes the major findings of the paper and outlines future work.

${ }^{1}$ http://www.openlivinglabs.eu/ 


\section{The TasLab network}

The TasLab initiative, being part of ENoLL, is a cooperation cluster supported by the government of the Autonomous Province of Trento, whose goal is to develop user-centric innovation involving all the stakeholders (citizens, public administrations, enterprises and research entities) by taking up actions to innovate the public services by funding targeted research projects on specific domains, investing in infrastructures (e.g., $800 \mathrm{~km}$ of fiber network), and adopting legislative ad-hoc actions. TasLab is articulated in a multidisciplinary eco-system driven approach where specific initiatives are identified within the domains that reflect the Trentino's vocation areas (e.g., eHealth, eInclusion, eEnvironment). Stakeholders that co-operate within the TasLab framework include actors with different levels of contribution, different goals, and different ways used to manage relations within the innovation process. We believe that such a diversity is not an issue but it is rather a value that should be handled properly in order to create the conditions for a successful integration of the innovation stakeholders in the Trentino territory [Giunchiglia, 2007]. The TasLab initiative (among others) has the goal of managing such a diversity, which acts as a driver to promote collaborative innovation and to build networks among the innovation stakeholders. In order to support these, a number of parallel key functions were identified, such as technology monitoring, screening and evaluation of the new technological concepts, market analysis, innovation management, dissemination, etc.

The objectives of the stakeholders. Public authorities need to continuously monitor and control the portfolio of the ongoing regional innovation projects as well as to have a clear view on the past projects with the perspective of building the new ones on top of the already obtained results and not to duplicate efforts. The interest is also to understand the social effects and to qualify the "social innovation balance" implied by the exploitation of the innovative tools, and to be able to measure the progress being made in terms of the structural, collective and social conditions for innovation in the region. Through the TasLab portal that includes sharing-concurrenttransparency functions, the public authorities can understand better (more precisely) the innovation status of the region, e.g., what are the concrete stakeholders that are more frequently involved in the innovation projects, what types of specific skills are growing, and to what extent these dynamics match the political interests and priorities. In general, public administrations look for innovative products and services for the local government by launching innovation programs and by trying to increase the internationalization of local SMEs through fostering new partnerships.

In turn, research centres aim at developing a more structured channel for technology transfer among academia and industry, at obtaining funds for future research activities, and so on. Finally, industries look for the new funding opportunities for the development of innovative solutions and aim at participating to the transformation of innovation into business value. Notice that the portal is designed to address directly the above mentioned objectives of the main innovation stakeholders; some further details on the SWOT analysis of the portal can be found in [Shvaiko et al., 2010].

On communication across the stakeholders. At present, the local government is supportive to the innovation activities and views research and innovation in ICT among the driving forces as the response to the crisis. In turn, this encourages the communication among the main innovation stakeholders and helps addressing more seriously the difficulties of finding a consensus among the various stakeholders by involving business companies, research centres and public entities in an integrated and transparent process. Such a process is subject to punctual monitoring and measurements by the local government in terms of innovation results, and hence the TasLab portal is envisaged to be a useful tool to support such a monitoring, in order to ultimately contribute and help the government shape the legislative actions to be respectively taken up. 


\section{The TasLab portal}

As previously discussed, a territorial innovation portal should provide knowledge management facilities in order to match all these different assets, interests, and competencies, as well as treat heterogeneity/diversity (e.g., in goals and languages) as a fundamental characteristic that generates innovation. In turn, this requires an adequate semantic heterogeneity management that we believe can be implemented with the help of the semantic technology.

We have realized various functionalities within the TasLab portal in order to foster collaborative innovation within the Trentino territory. The portal is available in English and Italian languages and it is implemented on top of the LifeRay ${ }^{2}$ portal server. It possesses various standard functionalities with public and private areas, including vision and mission of the initiative, related materials, news and events, services offered and so on. In Trentino there are about 350 entities, including various public administration departments and municipalities that are respectively structured, and which ultimately offer services to about 500.000 inhabitants, 13 research centres (grouping about 750 researchers) and 684 companies in ICT (grouping about 3.400 professionals) [Camussone et al., 2007]. Also, the system can be further extended, e.g., by adding research centres from across Europe. Notice that each of these actors develops its own business assets and knowledge that might enable innovation.

The main business entities involved in the Taslab portal are as follows. Organization, which can be of the following types: company, public entity (a final user in our case) and research center. User, which identifies a person who accesses the private area of the portal and uses the available functions (e.g., innovation tripole generation). Project, which can be created only by a user belonging at least to an organization which is directly associated to it. Tripole, which represents the group of organizations that have been involved in a project proposal.

The functionalities of the portal related to collaborative innovation include: semi-automatic innovation tripole generation ( $\$ 3.1$; which, to the best of our knowledge, is not available in any other portal), semantic search (§3.2), project proposal development with wiki and forum (§3.3), blog for an organization (§3.4). We describe here only those features that are peculiar of the TasLab portal. For each functionality mentioned above we show (i) the business need the functionality addresses (the problem); (ii) how users can exploit the functionality (the solution); (iii) technical details on the implementation. Some further technical details on the use of the semantic technology within the portal can be found in [Shvaiko et al., 2010].

\subsection{Innovation tripole generation}

Problem: the main stakeholders of the TasLab network expressed the need to facilitate collaborative innovation in the Trentino area. Specifically, the stakeholders communicated the lack of support to establish innovation collaborations among different organizations. In other words, even though the stakeholders might have good ideas about innovation, they typically do not have a complete picture on with whom they can actually collaborate.

Solution: the Taslab portal supports collaborative innovation via tripole generation, which allows for searching adequate competences in the context of a certain project idea. Such a functionality is available only to the registered users that are affiliated at least to one organization. The proponent user has to fill a project description form, a set of free text fields that define the project type, themes and principal technologies. The portal will then perform a search for business partners that can potentially collaborate with the user's organization in the proposed project. Possible partners are split according to their type (research, final users, and industry) and ranked on the basis of best-match criteria. If the proposed partnerships are compelling for the proponent, (s)he selects at least one partner for each partner type and invites them (via email) to join the consortium-to-be. For example, given a project theme, such as semantic geo-catalog, this

\footnotetext{
${ }^{2}$ http://www.liferay.com/
} 
functionality discovers the best match in terms of competences and interests across the innovation stakeholders by processing their profiles and their created content.

Technical details: the matching algorithm searches and matches competences at the semantic level. The metadata inserted into the system to characterize organizations is not bounded by any vocabulary or predefined lists of terms, thus, allowing users to use their own terminology which describes best their activities. Tripole generation considers not only organization descriptions, but also the contents generated by the users affiliated to the organizations.

\subsection{Semantic search}

Problem: a well known problem in the Information Technology field is that different stakeholders use different vocabularies. For instance, the vocabulary used by a researcher in computer science will be dramatically different from that used by a top-manager in a manufacturing company. In order to find relevant content (projects, organizations, documents) the vocabulary gap should be suitably bridged. Due to these vocabulary discrepancies, traditional syntactic matching does not work sufficiently well.

Solution: any registered user can pose queries by using a natural language format. Specifically, the user can choose any language supported by the portal (currently, English and Italian) and write free text queries. The portal processes the query by running semantic search algorithms that return the most relevant content related to the request. The portal ranks matching content according to relevancy criteria. The search engine scans a wide range of content: organizations' descriptions, documents uploaded on the portal (e.g., in doc, pdf), published content (blogs), etc. The user sees ranked results, filter them according to the category (organization, project, document), use keywords for granular filtering.

Technical details: the TasLab portal implements a semantic search functionality which acts as the portal search engine. It represents an improvement over the canonical (and commonly known) exact string matching search methods available in LifeRay. The portal exploits an enriched version of the Eurovoc ${ }^{3}$ ontology to guide semantic search. Eurovoc has been refined by adding specific terms that specialize the lower-level of the ontology. Semantic search also exploits stemming to reduce inflected and derived words to their root (stem), so that search is even more flexible.

\subsection{Project proposal development with wiki and forum}

Problem: writing a project proposal is a challenging and tricky task; many different people have to collaborate in order to set up a consistent and sound document. In the development of a proposal, different documents are exchanged, various sections of the proposal have to be written by different people, revisions and comments are daily activities. Once a consortium is defined, the challenge is how their collaboration can be effectively supported.

Solution: once a project consortium is stable, participants gain access to the project homepage where they can start discussing it using built-in wiki and forum functionalities, which provide immediate technical support for the development of a project proposal idea. The project concept can be collaboratively developed using the wiki: a draft structure of the proposal document is defined, then multiple people can edit it at the same time till the proposal satisfies the participants. Whenever the wiki discussion functions are not sufficient, the participants can exploit the forum module to reconcile viewpoints and address other problems. The wiki module also allows for uploading documents (e.g., state-of-the art, budget prospects, meetings' minutes). Users can compare the current wiki version with the previous ones by looking up the history, which shows older versions and specifies which user applied the modification. Visual comparison of versions is also possible. These features result in more effective and efficient work processes. For example, by monitoring what users are contributing and when they

\footnotetext{
${ }^{3}$ http://europa.eu/eurovoc/
} 
contribute such that a project manager can quickly verify if the assigned tasks were completed, with revision tracking, document polishing and data sharing becomes simpler.

Technical details: The wiki and the forum features are provided via a standard modules of LifeRay. These modules are quite complete, widely tested by large user communities, and currently maintained. Generated content analysed by the tripole generation module to identify collaboration opportunities via detection of competences.

\subsection{Blog for an organization}

Problem: any organization aims to enhance its communication towards customers, partners and general audience in order to increase its visibility. The traditional way to promote the organization is through its own institutional website. The main drawback of such an option is that small organizations will hardly manage to attract new visitors. Since the TasLab network is intended to enhance innovative collaboration among organizations, the TasLab portal is the adequate means for participating organizations to improve their visibility.

Solution: one of the main entities in the TasLab portal is that of organization. Organizations can be created by specific users (owners). Other users can join that organization on the portal upon affiliation approval by the owner. The organization is not just a description used for tripole generation, but it also enables its owner to post publicly visible content. The TasLab portal offers a built-in blog for business purposes, namely to enhance the communication within the portal as well as for marketing, or public relation purposes to inform portal members and other interested parties in the activities of an organization. Blogging features range from simple textual descriptions to multimedia elements. An organization owner can add descriptions of events, publish images, add links to other blogs. If the owner activates comments for specific posts, other users can add their opinions.

Technical details: As for the wiki and the forum, the blog feature is provided via a standard LifeRay module. All the information in blog posts and comments is used to enhance semantic search and tripole generation functionalities.

In summary, the Taslab portal provides technological support to business needs concerning collaborative innovation via Web 2.0 tools, such as wiki and blog, enhanced with the semantic technology enabling semantic search and innovation tripole semi-automatic generation. The features of the TasLab portal have been selected and implemented in a systematic way starting from the requirements expressed by the stakeholders. Such requirements helped us understand what are the business needs of the various stakeholders and identify those problems that have to be supported by the TasLab portal.

\section{Discussion}

The TasLab portal provides knowledge management facilities (e.g., competence matching) for collaborative innovation. The portal aims not only to model the pre-existing knowledge, but also to foster collaboration within public administrations, industrial companies and research centres by suitably managing heterogeneity and diversity of these actors. Specifically, the portal provides a combination of the best technologies currently available, producing new structured knowledge driven by user created content with the help of the semantic technology (e.g., Eurovoc-based semantic search) and Web 2.0 tools (e.g., wiki), thereby amplifying potential innovation gains by building on top of a larger volume of knowledge and experience expressed in terms of the user created content. This directly affects the business and the working environment by providing new opportunities, such as new user collaborations being accessible to all organizations subscribed to the portal, and thus, taking into account the concerns of the various innovation stakeholders scattered across Trentino and around the world. 
On sustainability of the portal usage. Our goal is that the portal becomes the reference point for the generation of the regional innovation projects. The system will allow the stakeholders to search over the competences available on the territory, to create collaboratively new innovative projects, to monitor and control project results and regional impacts. It will also provide visibility for companies, opportunities for business and (competence) growth, what is especially important for small and medium enterprises.

Anyhow, we expect to encounter some resistance with the tool usage based on cultural issues, what is typically happening with almost all knowledge management tools. The plan is to perform some concrete actions for educating front-line workers, such that they are recognized for sharing and reusing knowledge. Notice that this is not a technical effort, but rather a cultural endeavour. A suitably defined set of incentives and a reward program (not necessary monetary, e.g., with contests giving professional recognition, opportunities of advertising organizations' activities) will be designed. The communities of practice will be established to come up with success stories and with a critical mass of actionable knowledge. The resistance is going to be attacked by isolating such users and by addressing their issues one by one as pilots, and hence, delivering value through the pilots. This means being user-centric to the end, getting people involved step by step, refining the understanding of their needs, etc. We see this as an iterative process that should run until the portal does not become self-sustained. Finally, the major communications will be made quarterly across the stakeholders.

Implications on methodology and theory of innovation. The framework where we based our concept and where we evaluated the requirements for designing the tool to support local innovation is based on the paradigm of user-centric and open innovation. In this context the TasLab portal carries advanced features and functionalities that were designed with the actual users themselves, and yet preliminary tested in several different domains. We believe that the features that the portal provides can shift the paradigm used by innovation stakeholders to interact more with each other: the portal is no longer a place where to search for entities doing things and where to possibly find some useful competences or references by browsing organization profiles, but it rather becomes almost an innovation actor itself by managing the creation of networks and revealing hidden relations, which could not be discovered by singular users acting separately.

Implications on concurrent enterprising. The TasLab portal provides a technological support to generate new business opportunities and enables more efficient interactions (e.g., shorter and accelerated interaction cycles) among the key innovation stakeholders: public administrations, research centres/universities and industries. It helps interconnecting the know-how and competencies of the stakeholders by providing flexible ways of interactions (compared to an earlier enterprise paradigms) based on processing of the user defined content at the conceptual level with the help of the semantic technology, what we believe is adequate to the pace of the actual global market, hence, lowering the entrance barriers and helping small and medium enterprises to collaborate more fruitfully and develop innovative projects. Specifically, it accelerates tender/project proposal preparation by sharing and reusing knowledge of the involved partners. In fact, working concurrently at the early stages of a project proposal helps building consensus faster (by sharing concepts and knowledge, rather than information), and therefore, reduces global costs of the product- or service-to-be.

\section{Conclusions}

Living Labs are by definition multidisciplinary eco-systems structured around specific domains, where innovation management have to deal with the diversities that characterize the actors involved in the innovation process. In order to exploit such a diversity rather than trying to minimize it, suitable tools and operations are to be designed in order for the various stakeholders to share and reuse concepts and solutions. In this paper we have presented our experience with 
fostering collaborative innovation within the TasLab environment, by providing a portal that aims not only to model the pre-existing knowledge, but also to foster collaboration within public administrations, industrial companies and research centres by suitably managing the diversity of these actors.

The TasLab portal provides various functionalities, including semi-automatic innovation tripole generation from the user created content, semantic search for competences, and so on. We have currently only tested the effectiveness of the approach from a technical point of view, yielding promising results, e.g., in terms of the scalability of the portal. While a methodology for the comprehensive evaluation of the portal is currently under development, preliminarily identified measures here include (beyond the traditional activity monitoring): the number of successfully generated project networks, the number of projects delivered to production. We will also exploit some analytics to understand what types of users are using or not using knowledge, what types of knowledge, in what contexts, etc.

\section{Acknowledgement}

This work has been supported by the TasLab network project funded by the European Social Fund under the act $n$. 1637 (30.06.2008) of the Autonomous Province of Trento. We are thankful to the TasLab group members: Isabella Bressan, Ivan Pilati, Valentina Ferrari, Marco Combetto and to Fausto Giunchiglia, Paolo Giorgini, Roberta Cuel, Francesca Gleria, Italo Della Noce, Mauro Piffer and Andrea Simioni for many fruitful discussions on the various innovation aspects covered in this paper.

\section{References}

Ardilio A., Auernhammer K., \& Kohn S. (2004) Marktstudie Innovationssysteme: ITUnterstützung im Innovations management, Stuttgart, Germany: Fraunhofer IRB.

Camussone P.F., Dalmonego, I., \& Zaninotto, E. (2007) Le tecnologie digitali nell'economia del Trentino. EGEA S.p.A., Italy

Chesbrough H. W. (2003) Open Innovation: The New Imperative for Creating and Profiting from Technology. Harvard Business School Press.

Gassmann, O. \& Enkel, E. (2004) Towards a Theory of Open Innovation: Three Core Process Archetypes. In Proceedings of RADMA.

Giunchiglia F. (2007) The role of the research centers in the development of Trentino. In Le Tecnologie Digitali nell'economia del Trentino.

Eriksson M., Niitamo V.-P., Kulkki S., and Hribernik K. A. (2005) Living labs as a multi-contextual R\&D methodology. In Proceedings of ICE.

De Leon M.P., Eriksson M., Balasubramaniam S., Donnelly W. (2006) Creating a distributed mobile networking testbed environment through the Living Labs approach. In Proceedings of TRIDENTCOM.

Katzy B., Loeh H., Sung G. (2005) The CeTIM virtual enterprise lab - a living, distributed, collaboration lab. CeTIM working paper series, Working paper no 3305.

Lausen H., Ding Y., Stollberg M., Fensel D., Lara Hernandez R., and Han S.-K. (2005). Semantic web portals: state-of-the-art survey. Journal of Knowledge Management, 9(5):40-49.

Riedl C.; May, N.; Finzen, J.; Stathel, S.; Kaufman, V. \& Krcmar, H. (2009) An Idea Ontology for Innovation Management International. Journal on Semantic Web and Information Systems, 5(4), 1-18.

Shvaiko P., Oltramari A., Cuel R., Pozza D., Angelini G. (2010) Generating innovation with semantically enabled TasLab portal. In Proceedings of ESWC.

Van Gundy A. (1988) Techniques of structured problem solving. Van Nostrand Reinhold, USA.

Von Hippel E. (2005) Democratizing Innovation. MIT Press, USA. 